\title{
Análise de Teoria de Resposta ao Item de um Instrumento Breve de Avaliação de Comportamentos Antissociais
}

\author{
Nelson Hauck Filho \\ Universidade São Francisco \\ Itatiba, SP, Brasil \\ Roberta Salvador-Silva \\ Pontificia Universidade Católica do Rio Grande do Sul \\ Porto Alegre, RS, Brasil \\ Marco Antônio Teixeira \\ Universidade Federal do Rio Grande do Sul \\ Porto Alegre, RS, Brasil
}

\begin{abstract}
RESUMO
Comportamentos antissociais são comuns a diversas condições psicopatológicas, incluindo transtornos da personalidade (e.g., antissocial e narcisista) e transtornos do humor (e.g., transtorno bipolar). Todavia, até o momento, havia uma importante lacuna no contexto brasileiro no que diz respeito à avaliação breve dos comportamentos antissociais em indivíduos adultos de contextos não carcerários. Em virtude disso, o presente estudo teve como objetivo a construção e a análise mediante Teoria de Resposta ao Item de um instrumento breve para uso em pesquisas e rastreio junto à população geral adulta. As análises das respostas de 204 estudantes universitários (média de idades=23,56 anos; $D P=7,70 ; 60,6 \%$ mulheres) a um conjunto de itens permitiram reter 13 itens com excelentes propriedades psicométricas. Esses itens se mostraram avaliativos de um fator geral de antissocialidade, interpretável como uma propensão ao antagonismo, à não cooperação e à agressão em uma diversidade de contextos sociais. Limitações do estudo são discutidas ao final.
\end{abstract}

Palavras-chave: Comportamento antissocial. Personalidade. Teoria de Resposta ao Item. Análise fatorial confirmatória.

\begin{abstract}
Item Response Theory Analysis of a Brief Instrument for Assessing Antisocial Behaviors

Antisocial behaviors co-occur with several psychopathological conditions, such as personality disorders (e.g., antisocial, narcissistic) and affective disorders (e.g., bipolar disorder). Nevertheless, up to now Brazilian researchers have dealt with an important methodological shortcoming, as we found no available brief instruments for assessing antisocial behaviors of non-prisoner Brazilian adult individuals. Therefore, the present study had the aim of constructing and performing Item Response Theory analyses of a brief self-report instrument designed for use with adult individuals from the Brazilian general population. Analyses of data collected from 204 undergraduate students $($ mean age $=23.56$ years; $S D=7.70 ; 60.6 \%$ females) yielded a 13-item unidimensional instrument with excellent psychometric properties. Items provided information on a general factor that may be understood as a propensity toward antagonism, non-cooperation, and aggression in a great diversity of social contexts. We further discuss limitations of the study and make suggestions for future investigations.
\end{abstract}

Keywords: Antisocial behaviors. Personality. Item Response Theory. Confirmatory factor analysis.

\section{RESUMEN}

\section{Análisis de Teoría de Respuesta al Ítem de un Instrumento Breve Para la Evaluación de Conductas Antisociales}

Conductas antisociales son comunes a varias condiciones psicopatológicas, incluyendo trastornos de la personalidad (e.g., antisocial y narcisista) y los trastornos del estado de ánimo (e.g., trastorno bipolar). Sin embargo, hasta ahora, había la necesidad de instrumentos breves de evaluación de la conducta antisocial de adultos de la población general adulta brasileña. Como resultado de ello, el objetivo del presente estudio fue la construcción y el análisis usando la Teoría de Respuesta de un instrumento breve de conductas antisociales para uso en la población general adulta. El análisis de las respuestas de 204 estudiantes universitarios (edad media=23,56 años, SD=7,70; 60,6\% mujeres), resultó en un conjunto de 13 ítems con excelentes propiedades psicométricas. Estos ítems fueron explicados por un factor general de antisocialidad, interpretable como una propensión al antagonismo, la falta de cooperación y la agresión en una variedad de contextos sociales. Las limitaciones del estudio se discuten al final.

Palabras clave: Comportamiento antisocial. Personalidad. Teoría de Respuesta al Ítem. Análisis factorial confirmatorio. 
Comportamentos antissociais são violações intencionais de normas implícitas ou explícitas que são relevantes para a cultura do indivíduo (Burt e Donnellan, 2008). Esses comportamentos envolvem tanto aspectos sutis de falta de reciprocidade nas interações interpessoais quanto comportamentos de natureza agressiva, sendo explicados por múltiplas trajetórias desenvolvimentais (Frick e Viding, 2009). Comportamentos socialmente desviantes são comuns a diversas condições psicopatológicas; por exemplo, ocupam um papel central no Transtorno da Personalidade Antissocial (American Psychiatric Association, 2013), e são também correlatos de traços de psicopatia (Cooke, Michie, Hart e Clark, 2004), e de Transtorno da Personalidade Borderline (Chabrol, Valls, Leeuwen e Bui, 2012), Transtorno da Personalidade Narcisista (Reidy, Zeichner, Foster e Martinez, 2008), e Transtorno Bipolar (Swann et al., 2011), além de outras categorias nosográficas. De um ponto de vista neurobiológico, comportamentos antissociais, especialmente aqueles agressivos, são influenciados por uma baixa ativação das estruturas cerebrais frontais (e.g., orbitofrontal) e das amígdalas (Blair, 2004), bem como por uma alta razão testosterona/cortisol (Terburg, Morgan e Van Honk, 2009).

No que diz respeito à avaliação dos comportamentos antissociais, uma das estratégias cada vez mais empregadas na literatura são os instrumentos de autorrelato (Burt e Donnellan, 2009). No contexto nacional, os principais instrumentos disponíveis são: o Cuestionario de Conducta Antisocial (CCA; Luengo, Otero, Romero, Gómez-Fraguela e TavaresFilho, 1999; 70 itens distribuídos nas subescalas vandalismo, agressão, roubo, conduta contra normas e uso de drogas); a Escala de Condutas Antissociais e Delitivas (ECAD; Formiga, 2003; 40 itens que compõem as subescalas antissocial e delitiva); o Questionário de Comportamentos Antissociais e Delitivos (CAD; Gouveia, Santos, Pimentel, Diniz e Fonseca, 2009; uma versão de 20 itens do instrumento da Escala de Condutas Antissociais e Delitivas); a Escala de Avaliação de Tendência à Agressividade (EATA; Sisto; Cecilio-Fernandes e Silveira, 2012; 40 itens avaliativos de comportamentos agressivos, dissimulação, manipulação, persuasão pela mentira, intimidação pela chantagem e vingança); e a Escala de Conduta Social (ECS; Reppold, 2005; 80 itens que formam as subescalas antissocial, desafio-oposição e prossocial).

Todos esses instrumentos apresentam propriedades psicométricas aceitáveis para a avaliação de comportamentos antissociais. Contudo, algumas características podem restringir a sua aplicação no contexto da pesquisa. O número de itens do instrumento, por exemplo, pode representar uma limitação devido ao tempo de aplicação requerido, de modo que uma grande quantidade de itens pode indispor os voluntários a participarem do estudo. Nesse sentido, medidas mais breves podem apresentar vantagens em baterias de avaliação que contenham muitos instrumentos ou em situações de triagem (Hauck Filho, Machado, Teixeira e Bandeira, 2012). Por um lado, a CAD (Gouveia et al., 2009) é o único dos instrumentos brasileiros com aplicação breve (possui 20 itens). Por outro lado, a CAD apresenta itens com conteúdo mais direcionados para crianças e adolescentes (e.g., "Tocar a campainha na casa de alguém e sair correndo" e "Apanhar frutas em um jardim ou pomar alheio"), não sendo muito adequada para a avaliação de adultos. Ainda outra dificuldade metodológica diz respeito ao foco dos instrumentos em comportamentos antissociais predominantemente criminosos. Essa ênfase traz vantagens para a aplicação em contextos forenses, mas dificulta sua aplicação na população geral (e.g., estudos com estudantes universitários).

Em virtude disso, seria útil a pesquisadores brasileiros a disponibilidade de um instrumento breve e de autorrelato que enfatizasse comportamentos antissociais encontrados em indivíduos adultos da população geral. De fato, a avaliação da antissocialidade em contextos não-forenses e não prisionais se sustenta na dimensionalidade dos aspectos latentes subjacentes aos indicadores de comportamentos antissociais (Walters, 2009). De fato, um fator geral de antissocialidade já foi sugerido como explicativo da covariância entre os critérios diagnósticos para Transtorno da Personalidade Antissocial (Marcus, Lilienfeld, Edens e Poythress, 2006) e entre os fatores do instrumento Psychopathy Checklist-Revised (Patrick, Hicks, Nichol e Krueger, 2007). Analogamente, Walters (2008) hipotetizou a existência de uma hierarquia de traços de antissocialidade cuja ordem superior seria composta por um fator geral (e cuja ordem inferior seria representada por tendências reativas e instrumentais). Esse fator geral da antissocialidade representaria uma propensão a se comportar de maneira antagonista, desviante e agressiva, sendo que a localização do indivíduo no contínuo da variável influenciaria na frequência com que apresenta esse tipo de conduta em uma série de contextos sociais.

O objetivo do presente estudo foi a construção e a análise psicométrica de um instrumento breve de autorrelato para a avaliação de comportamentos antissociais em indivíduos adultos e adultos jovens da população brasileira. Os itens do instrumento 
foram planejados de modo que apresentassem um nível de dificuldade compatível com a população geral, ou seja, que não representassem aspectos extremos somente observáveis em contextos carcerários. Além disso, as estratégias analíticas utilizadas, uma combinação de métodos de análise fatorial categórica e Teoria de Resposta ao Item (TRI), buscaram reter apenas itens maximamente discriminativos do traço latente avaliado. Esses métodos levaram em consideração a natureza ordinal da escala Likert utilizada, tendo em vista possíveis vieses decor rentes de não atentar para esse aspecto (HolgadoTello, Chacón-Moscoso, Barbero-García e Vila-Abad, 2010).

\section{MÉTODO}

\section{Participantes e procedimentos}

Os participantes do estudo foram 204 estudantes de uma universidade federal do Rio Grande do Sul com idades entre 17 e 54 anos (média $=23,56 ; D P=7,70$ ), sendo $60,6 \%$ mulheres. As coletas foram feitas em salas de aula, após permissão de professores e de assinaturas de termos de consentimento pelos participantes do estudo. O presente trabalho integra um estudo mais amplo, cujo projeto foi previamente aprovado pelo Comitê de Ética em Psicologia da Universidade Federal do Rio Grande do Sul (UFRGS).

\section{Instrumento}

Foram elaborados 24 itens para avaliar diversos tipos de comportamentos antissociais. Os itens foram elaborados a partir dos seguintes construtos teóricos: agressão reativa (i.e., agressão motivada por raiva e outros afetos negativos, Miller e Lynam, 2006; e.g., “Quebra coisas quando está enraivecido?”), agressão instrumental (i.e., agressão como meio para obter recompensas, Miller e Lynam, 2006; e.g.,"Machuca alguém para conseguir algo que quer muito?"), agressão indireta (i.e., exclusão social e difamação, Burt e Donnellan, 2009; e.g., "Inventa coisas para manchar a reputação de alguém?") e violações de regras (i.e., conduta antagonista e intencionalmente desviante, Burt e Donnellan, 2009; e.g., "Não cumpre, de propósito, com um acordo feito com outra pessoa?"). Cuidou-se para que os itens representassem situações que não fossem tão extremas a ponto de serem endossados apenas por indivíduos de contextos prisionais e forenses. $\mathrm{O}$ foco foram comportamentos antissociais predominantemente (embora não exclusivamente) não criminosos. A escala de resposta utilizada foi $1=$ Nunca, $2=$ Algumas vezes, $3=$ Diversas vezes $\mathrm{e}$ $4=$ Quase sempre ou sempre.

\section{Análise dos dados}

A dimensionalidade do instrumento foi investigada com o método Hull Confirmatory Fit Index (LorenzoSeva, Timmerman e Kiers, 2011), a partir de uma análise fatorial exploratória categórica Unweighted Least Squares (ULS) com correlações policóricas. De acordo com o estudo de simulação de LorenzoSeva et al. (2011), o método Hull é um dos melhores métodos disponíveis para estimar a dimensionalidade de um conjunto de itens. A análise foi conduzida com o software Factor 9.2 (Lorenzo-Seva e Ferrando, 2006). $\mathrm{Na}$ sequência, o conjunto final de itens foi avaliado a partir de uma análise fatorial confirmatória categórica Weighted Least Squares Mean- and Variance-adjusted (WLSMV; Muthén, Du Toit e Spisic, 1997) e por meio do modelo de TRI Graded Response (GRM; Samejima, 1969). Embora ambos os métodos sejam equivalentes, sua utilização conjunta teve por objetivo principal obter a informação da discriminação dos itens tanto na métrica tradicional da carga fatorial padronizada (WLSMV) quanto do parâmetro $a$ em escala logística (GRM). A análise WLSMV foi conduzida com o programa Mplus 6.12 (Muthén e Muthén, 2010), enquanto a análise de TRI foi feita com o pacote ltm (Rizopoulos, 2006) do software R. Todos os métodos de análise foram planejados para levar em consideração o fato de que escalas Likert utilizada são compostas de categorias ordenadas, não sendo possível assumi-las como um variáveis contínuas (Holgado-Tello et al., 2010).

\section{RESULTADOS}

Os dados se mostraram adequados para a análise fatorial exploratória ordinal, $\mathrm{KMO}=0,93, \chi^{2}$ de Bartlett $=2384,0, p<0,001$. A análise de dimensionalidade pelo método Hull Comparative Fit Index (CFI) sugeriu uma solução unidimensional como mais parcimoniosa. Tendo em vista o objetivo de reter um conjunto reduzido de itens maximamente discriminativos, foram excluídos itens com cargas fatoriais abaixo de 0,50 e comunalidades abaixo de $25 \%$. Disso, resultou um conjunto de 13 itens, sendo que o primeiro autovalor se mostrou explicativo de $66,94 \%$ da variância total dos itens. Esses itens apresentaram poucas correlações residuais $(\mathrm{RMSR}=0,038)$, indicando que foi satisfeito o pressuposto da independência local do modelo de análise fatorial; ou seja, as correlações entre os itens foram próximas a 0,0 , após controlar a variância explicada pelo fator.

O conjunto final de 13 itens foi composto por itens unidimensionais e altamente discriminativos da 
variável latente. Esses itens foram então avaliados mediante a análise fatorial confirmatória categórica WLSMV e o modelo GRM. Os resultados dessas análises são apresentados na Tabela 1. O modelo apresentou um excelente ajuste, $\chi^{2}=77,31, g l=65$, $p=0,141, \mathrm{CFI}=0,998, \mathrm{TLI}=0,997, \mathrm{RMSEA}=0,030$ (IC $90 \%=0,000-0,054)$. Conforme se pode ver na Tabela 1, foi bastante alta a discriminação de todos os itens, tal como vista a partir das cargas fatoriais ou a partir dos parâmetros $a$ do GRM. Outro ponto relevante é que os parâmetros da dificuldade dos itens indicaram que os itens abrangeram uma ampla porção do traço latente, entre $-0,22$ e 3,80.

Por fim, avaliou-se a fidedignidade e a quantidade de informação psicométrica proporcionada pelos itens. Considerando os itens como contínuos, os coeficientes alpha de Cronbach e Lambda2 de Guttman resulta- ram ambos em um valor de 0,92. Por outro lado, ao considerar os itens meramente como ordinais, o coeficiente alpha de Cronbach foi de 0,97, tal como calculado a partir do software Factor 9.2. Foram também inspecionadas as curvas de informação dos itens e do teste. Essas curvas permitem, ao contrário dos tradicionais coeficientes de fidedignidade, investigar, em profundidade, em quais regiões do traço latente o teste é mais preciso e apresenta menor erro para estimar a localização dos indivíduos. Os 13 itens forneceram um total de 65,77 pontos de informação acerca de todo o contínuo do traço latente, tendo sido mais informativos no intervalo entre 0,00 e 4,00, como apresentado na Figura 1. O ponto 0,00 representa a média da distribuição do traço latente, o que significa que os itens estiveram localizados na porção superior da variável latente.

TABELA 1

Análise fatorial confirmatória WLSMV (cargas fatoriais padronizadas) e Graded Response Model (discriminação e dificuldade)

\begin{tabular}{|c|c|c|c|c|c|}
\hline & $\begin{array}{c}\text { Carga } \\
\text { fatorial }\end{array}$ & $a$ & $b_{1}$ & $b_{2}$ & $b_{3}$ \\
\hline 1. Tenta enganar colegas de trabalho ou estudo para obter vantagens? & 0,81 & 2,00 & 0,94 & 2,56 & 3,80 \\
\hline 2. Mente para causar uma boa impressão? & 0,68 & 1,54 & $-0,16$ & 2,02 & 3,58 \\
\hline 3. Não cumpre, de propósito, com um acordo feito com outra pessoa? & 0,89 & 2,34 & 0,76 & 1,91 & - \\
\hline 4. Machuca alguém para conseguir algo que quer muito? & 0,94 & 3,54 & 1,08 & 1,94 & 3,50 \\
\hline 5. Pega coisas de outras pessoas sem pedir emprestado? & 0,67 & 1,50 & 0,28 & 1,94 & 3,50 \\
\hline 6. Machuca animais intencionalmente? & 0,88 & 2,47 & 1,29 & 1,94 & 2,77 \\
\hline 7. Inventa coisas para manchar a reputação de alguém? & 0,92 & 3,50 & 1,27 & 1,88 & 2,50 \\
\hline 8. Culpa outras pessoas por coisas que você fez? & 0,84 & 2,65 & 0,45 & 1,78 & 2,68 \\
\hline 9. Faz ameaças a outras pessoas para conseguir o que quer? & 0,81 & 2,32 & 0,94 & 1,92 & 2,68 \\
\hline 10. Usa nomes falsos para não ser responsabilizado por algo de errado que fez ou pretende fazer? & 0,84 & 2,39 & 1,23 & 1,92 & 2,50 \\
\hline 11. Pratica atos de vandalismo? & 0,85 & 2,25 & 1,23 & 2,00 & 3,16 \\
\hline 12. Reage agressivamente a provocações? & 0,63 & 1,30 & $-0,22$ & 1,57 & 3,06 \\
\hline 13. Faz algo para se vingar de alguém que você considera que ofendeu ou magoou você?? & 0,71 & 1,50 & 0,02 & 1,70 & 2,62 \\
\hline
\end{tabular}

Curva de Informação do item

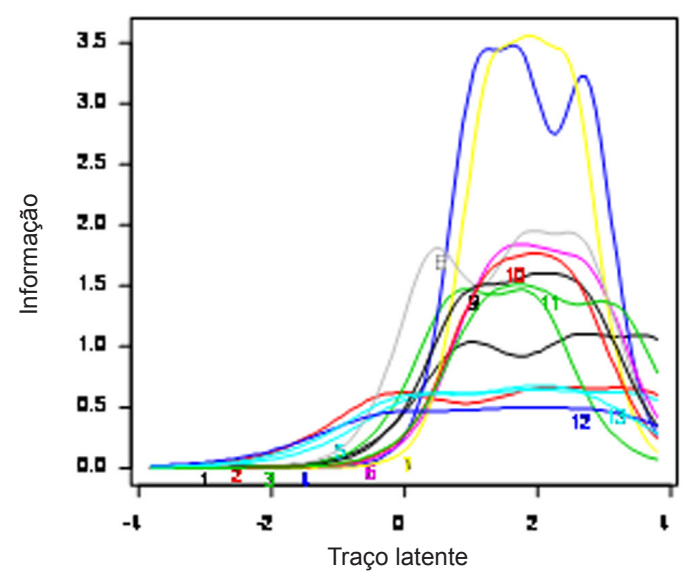

Função de Informação do teste

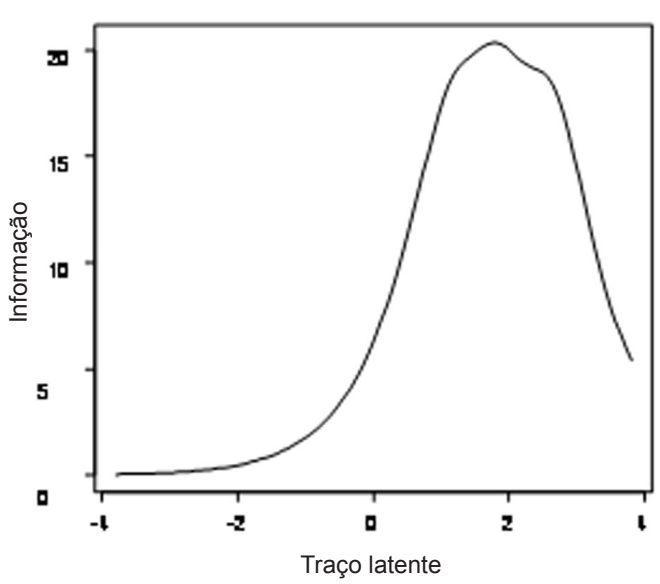

Figura 1. Curvas de informação dos itens e função de informação total do teste 


\section{DISCUSSÃO}

O objetivo do presente estudo foi desenvolver um instrumento de autorrelato avaliativo de comportamentos antissociais, bem como investigar as suas propriedades psicométricas. Os resultados revelaram um excelente ajuste para um modelo unidimensional composto de um conjunto de 13 itens de autorrelato. Todos os itens do instrumento se mostraram altamente discriminativos (i.e., altos valores para cargas fatoriais e parâmetros $a$ ), o que sugere uma adequada capacidade de diferenciar indivíduos localizados em regiões distintas da variável latente. Apesar da reduzida quantidade de itens, a curva de informação do teste e a análise de consistência interna evidenciaram bons índices de fidedignidade e de informação proporcionada pelo instrumento. Houve uma ampla cobertura do traço latente pelos itens, ainda que com um nível de precisão maior para a porção $50 \%$ superior da distribuição do traço latente.

A análise exploratória ULS com o método Hull e a análise confirmatória WLSMV forneceram suporte empírico para um modelo unidimensional reflexivo de comportamentos antissociais. Um modelo reflexivo é aquele em que a direção causal teórica é no sentido variável latente $\rightarrow$ itens (Bollen e Lennox, 1991). Nesse sentido, os itens se mostraram avaliativos de um contínuo latente interpretável como uma propensão geral a se comportar de maneira agressiva e antissocial em uma variedade de contextos, incluindo o acadêmico ou profissional, a família e os relacionamentos interpessoais em geral. Portanto, embora ainda seja necessário correlacionar o instrumento com avaliações externas de comportamento antissocial, os achados sugerem que o presente instrumento proporciona informação sobre esse possível fator geral descrito na literatura (Marcus et al., 2006).

$\mathrm{O}$ modelo fatorial reflexivo do instrumento significa que, quanto mais alto o escore do indivíduo no instrumento (escore latente estimado via TRI ou escore bruto), maior é a probabilidade de apresentar antagonismo, baixa cooperação, hostilidade e agressão em diversas situações. A presença de itens localizados na porção superior do contínuo da variável sugere que o instrumento, apesar de ter sido desenvolvido para uso, primariamente, com a população geral, resulta ser também potencialmente útil para a aplicação na população carcerária. Novos estudos, todavia, são necessários para investigar a sua adequação nesses contextos. Outro aspecto que também deve ser mais bem avaliado é a invariância dos parâmetros dos itens ao longo de grupos populacionais específicos, como grupos étnicos, regiões do país e sexo biológico (ou gênero).
Outro aspecto que vale ressaltar é que o planejamento do estudo foi feito de modo a utilizar análises estatísticas adequadas à natureza ordinal dos dados. Por exemplo, a avaliação da dimensionalidade do instrumento a partir de uma matriz de correlações policóricas reduz a probabilidade de encontrar fatores espúrios decorrentes de níveis de dificuldade variáveis entre os itens (Holgado-Tello et al., 2010). Além disso, o presente estudo ilustra como métodos de análise fatorial categórica, como o modelo unidimensional estimado via WLSMV são equivalentes à análise de TRI GRM para itens politômicos. É fácil verificar, nesse sentido, que as cargas fatoriais apresentadas na Tabela 1 fornecem a mesma informação que os parâmetros da discriminação $a$, apenas em uma escala distinta. Nesse sentido, autores consideram que a análise fatorial de indicadores categóricos é indistinta da análise de TRI (Wirth e Edwards, 2007). De fato, a análise fatorial confirmatória categórica com estimação WLSMV oferece também estimativas dos thresholds ou dificuldades das categorias da escala (em escala probit), exatamente como o modelo GRM (em escala logística ou logit). Apenas priorizou-se, neste estudo, as estimativas paramétricas disponíveis para o GRM, por ser mais comum a interpretação de seus valores em escala logística.

\section{CONSIDERAÇÕES FINAIS}

São limitações do estudo o reduzido tamanho amostral e a composição não aleatória da amostra, que impossibilitaram a investigação do funcionamento diferencial dos itens quanto a variáveis sociodemográficas como o sexo e a etnia. Outro aspecto diz respeito a quão bem o fator geral identificado coincide com o fator geral de antissocialidade avaliado pelos critérios do Transtorno da Personalidade Antissocial. Nesse sentido, novos estudos devem ser conduzidos para se inspecionar a validade convergente do instrumento com avaliações clínicas do transtorno. Não obstante, os resultados preliminares aqui apresentados sugerem que o novo instrumento possui propriedades psicométricas excelentes, sendo um recurso útil para pesquisadores e profissionais envolvidos na avaliação dos comportamentos antissociais em indivíduos adultos. $\mathrm{O}$ instrumento breve de autorrelato apresentado pode suprir uma lacuna no que diz respeito aos recursos metodológicos disponíveis na Brasil para a avaliação de aspectos desviantes na população adulta.

Sugere-se que a análise taxométrica seja empregada, futuramente, para que sejam testados os modelos concorrentes taxônico e dimensional para os 
13 itens do instrumento. A investigação taxométrica, nesse sentido, pode fortalecer a interpretação da variável latente avaliada pelo instrumento como sendo um fator geral representativo de uma propensão ao comportamento antissocial.

\section{REFERÊNCIAS}

American Psychiatric Association. (2013). Diagnostic and Statistical Manual of Mental Disorders - DSM-5 (5 $5^{\text {th }}$ ed.) (p. 991). Washington: American Psychiatric Publishing.

Blair, R. J. R. (2004). The roles of orbital frontal cortex in the modulation of antisocial behavior. Brain and Cognition, 55(1), 198-208. doi:10.1016/S0278-2626(03)00276-8

Bollen, K., \& Lennox, R. (1991). Conventional Wisdom on Measurement: A Structural Equation Perspective. Psychological Bulletin, 110(2), 305-314.

Burt, S. A., \& Donnellan, M. B. (2008). Personality correlates of aggressive and non-aggressive antisocial behavior. Personality and Individual Differences, 44(1), 53-63. doi:10.1016/j.paid. 2007.07.022

Burt, S. A., \& Donnellan, M. B. (2009). Development and validation of the Subtypes of Antisocial Behavior Questionnaire. Aggressive behavior, 35(5), 376-398. doi:10.1002/ab.20314

Chabrol, H., Valls, M., Leeuwen, N. van, \& Bui, E. (2012). Callousunemotional and borderline traits in nonclinical adolescents: Personality profiles and relations to antisocial behaviors. Personality and Individual Differences, 53(8), 969-973. doi: 10.1016/j.paid.2012.07.017

Cooke, D. J., Michie, C., Hart, S. D., \& Clark, D. (2004). Reconstructing psychopathy: Clarifying the significance of antisocial and socially deviant behavior in the diagnosis of psychopathic personality discorder. Journal of Personality Disorders, 18, 337-357.

Frick, P. J., \& Viding, E. (2009). Antisocial behavior from a developmental psychopathology perspective. Development and psychopathology, 21(4), 1111-1131. doi:10.1017/S0954579409990071

Hauck Filho, N., Machado, W. de L., Teixeira, M. A. P., \& Bandeira, D. R. (2012). Evidências de validade de marcadores reduzidos para a avaliação da personalidade no modelo dos cinco grandes fatores. Psicologia: Teoria e Pesquisa, 28(4), 417-423. doi:10.1590/S0102-37722012000400007

Holgado-Tello, F. P., Chacón-Moscoso, S., Barbero-García, I., \& Vila-Abad, E. (2010). Polychoric versus Pearson correlations in exploratory and confirmatory factor analysis of ordinal variables. Quality \& Quantity, 44(1), 153-166. doi:10.1007/ s11135-008-9190-y

Lorenzo-Seva, U., \& Ferrando, P. J. (2006). FACTOR: a computer program to fit the exploratory factor analysis model. Behavior research methods, 38(1), 88-91.

Lorenzo-Seva, U., Timmerman, M. E., \& Kiers, H. A. L. (2011). The Hull Method for Selecting the Number of Common Factors. Multivariate Behavioral Research, 46(2), 340-364. doi:10.1080/ 00273171.2011 .564527

Marcus, D. K., Lilienfeld, S. O., Edens, J. F., \& Poythress, N. G. (2006). Is antisocial personality disorder continuous or categorical? A taxometric analysis. Psychological medicine, 36(11), 1571-1581. doi:10.1017/S0033291706008245

Miller, J. D., \& Lynam, D. R. (2006). Reactive and proactive aggression: Similarities and differences. Personality and
Individual Differences, 41(8), 1469-1480. doi:10.1016/j.paid. 2006.06.004

Muthén, B., Du Toit, S. H. C., \& Spisic, D. (1997). Robust inference using weighted least squares and quadratic estimating equations in latent variable modeling with categorical and continuous outcomes. Non-published technical report.

Muthén, L. K., \& Muthén, B. O. (2010). Mplus: Statistical analysis with latent variables. User's guide. Los Angeles: Muthén \& Muthén.

Patrick, C. J., Hicks, B. M., Nichol, P. E., \& Krueger, R. F. (2007). A bifactor approach to modeling the structure of the psychopathy checklist-revised. Journal of personality disorders, 21(2), 118-41. doi:10.1521/pedi.2007.21.2.118

Reidy, D. E., Zeichner, A., Foster, J. D., \& Martinez, M. A. (2008). Effects of narcissistic entitlement and exploitativeness on human physical aggression. Personality and Individual Differences, 44(4), 865-875. doi:10.1016/j.paid.2007.10.015

Reppold, C. T. (2005). Construção, validaçao e normatizaçao de uma bateria de cinco escalas para avaliaçao de ajustamento psicológico em adolescentes. Universidade Federal do Rio Grande do Sul.

Rizopoulos, D. (2006). ltm: An R package for latent variable modelling and item response theory analyses. Journal of Statistical Software, 17(5), 1-25.

Samejima, F. (1969). Estimation of latent trait ability using a response pattern of graded scores. Psychometrika Monograph Supplement No. 17, 34(4, Pt. 2).

Swann, A. C., Lijffijt, M., Lane, S. D., Kjome, K. L., Steinberg, J. L., \& Moeller, F. G. (2011). Criminal conviction, impulsivity, and course of illness in bipolar disorder. Bipolar disorders, 13(2), 173-181. doi:10.1111/j.1399-5618.2011.00900.x

Terburg, D., Morgan, B., \& Van Honk, J. (2009). The testosteronecortisol ratio: A hormonal marker for proneness to social aggression. International journal of law and psychiatry, 32(4), 216-223. doi:10.1016/j.ijlp.2009.04.008

Walters, G. D. (2008). Self-Report Measures of Psychopathy, Antisocial Personality, and Criminal Lifestyle Testing and Validating a Two-Dimensional Model. Criminal Justice and Behavior, 35(12), 1459-1483. doi:10.1177/0093854808320922

Walters, G. D. (2009). Latent Structure of a Two-Dimensional Model of Antisocial Personality Disorder: Construct Validation and Taxometric Analysis. Journal of Personality Disorders, 23(6), 647-660. doi:10.1521/pedi.2009.23.6.647

Wirth, R. J., \& Edwards, M. C. (2007). Item factor analysis: current approaches and future directions. Psychological methods, 12(1), 58-79. doi:10.1037/1082-989X.12.1.58

\section{Autores:}

Nelson Hauck Filho - Professor no Programa de Pós-Graduação Stricto Sensu em Psicologia da Universidade São Francisco.

Roberta Salvador-Silva - Doutoranda em Psicologia, área de concentração Cognição Humana, pela Pontifícia Universidade Católica do Rio Grande do Sul.

Marco Antônio Pereira Teixeira - Professor Adjunto do Programa de Pós-Graduação em Psicologia, Universidade Federal do Rio Grande do Sul.

\section{Endereço para correspondência:}

Roberta Salvador Silva

Av. Ipiranga, 6681 - Prédio 11, sala 925 - Partenon

CEP 90619-900 Porto Alegre, RS, Brasil

E-mail: robertasalvador.s@gmail.com

Recebido em: 28.8.2013.

Aceito em: 23.09.2013. 